Cite this: DOI: $10.1039 / \mathrm{c} 6 \mathrm{nr} 01122 \mathrm{e}$

\title{
Photonic hybrid crystals constructed from in situ host-guest nanoconfinement of a light-emitting complex in metal-organic framework pores $\uparrow$
}

\author{
Abhijeet K. Chaudhari, Matthew R. Ryder and Jin-Chong Tan*
}

We report the concept underpinning the facile nanoconfinement of a bulky luminous guest molecule in the pores of a metal-organic framework (MOF) host, which yields a hybrid host $\supset$ guest nanomaterial with tunable opto-electronic characteristics and enhanced photostability. Utilizing an in situ host-guest confinement strategy enabled by molecular self-assembly, we show that the highly emitting ZnQ [Zn(bis-8-hydroxyquinoline)] guest complexes could be rapidly encapsulated within the sodalite nanocages of zeolitic imidazolate framework (ZIF-8) host crystals. The nature of optical and electronic transitions phenomena of the guest-encapsulated ZIF- $8 \supset$ ZnQ has been elucidated by means of fluorescence and absorption spectroscopy measurements, and substantiated further via theoretical molecular orbital calculations revealing the plausible host-guest charge transfer mechanism involved. Evidence suggests that its photophysical properties are not only strongly determined by the host-guest co-operative bonding interactions within the environment of the confined MOF nanocage, but also can be engineered to manipulate its emission color chromaticity or to shield light-sensitive emitting guests against rapid photochemical degradation.

Received 7th February 2016, Accepted 23rd February 2016

DOI: 10.1039/c6nr01122e

www.rsc.org/nanoscale

\section{Introduction}

Light-emitting materials ${ }^{1}$ are one of the fastest expanding areas of functional materials research by virtue of their vast range of practical applications, ranging from energy-saving LEDs $^{2}$ for lighting and display technologies, to photonic sensors, ${ }^{3-5}$ fluorescent switches, ${ }^{6,7}$ and bio-imaging devices. ${ }^{8}$ Particularly the development of solid-state luminescent materials has attracted an enormous interest, reflecting the major growth experienced by the portable consumer electronics industry. ${ }^{9}$ From the perspective of new optical materials, conjugated organic molecular assemblies, ${ }^{1}$ rareearth complexes, ${ }^{10}$ inorganic compounds, ${ }^{11,12}$ and hybrid inorganic-organic frameworks ${ }^{13}$ are amongst the most extensively studied today. Primarily this is because of their attractive opto-electronic properties arising from the rich structural and chemical versatility of such diverse systems, encompassing tunable molecular moieties of electron-rich functional groups and a wide choice of metal centers.

An exciting topic in the field of light-emitting materials concerns the bottom-up fabrication of opto-electronic devices integrating materials with controlled architectures and

Department of Engineering Science, University of Oxford, Parks Road, Oxford OX1 3PJ, UK. E-mail: jin-chong.tan@eng.ox.ac.uk

$\dagger$ Electronic supplementary information (ESI) available. See DOI: 10.1039/ c6nr01122e ordered microstructures, ${ }^{14,15}$ which are fundamentally important to effect unidirectional light production, inter-/intra-molecular electron transport, efficient light conversion and energy harvesting. Among the most promising contemporary materials featuring a highly-ordered crystalline network are the hybrid (inorganic-organic) coordination compounds, ${ }^{13,16}$ known as metal-organic frameworks (MOFs) or porous coordination polymers (PCPs). ${ }^{17-19}$ MOFs or PCPs possess two active sites to tune their opto-electronic properties, viz. organic linkers and metal centers. In principle by varying these two basic building blocks, the optical and electronic properties of the compound can be manipulated. ${ }^{20,21}$ Furthermore, their nanoporous framework architectures offer active coordination voids (via long-range ordered nanocages) which could establish complex intermolecular interactions ${ }^{22,23}$ and, by accommodating functional guest molecules to tune energy transfer and electronic conductivity. ${ }^{24}$

There are many recent studies on luminescent MOFs ${ }^{25-28}$ that elaborated on a number of promising methodologies to afford encapsulation of light-emitting metal complexes either as a linker ${ }^{29,30}$ or as a guest, ${ }^{31}$ for instance: cationic dyes, ${ }^{32-34}$ quantum dots, ${ }^{35,36}$ nanoparticles, ${ }^{37,38}$ or small luminous molecules $^{39}$ within the MOFs for manipulating its light emission behavior and photophysical properties. In fact the guestinduced properties of MOFs is an emerging area that could yield numerous novel device applications. ${ }^{20,24}$ At present, however, there are at least two major constraints encountered 


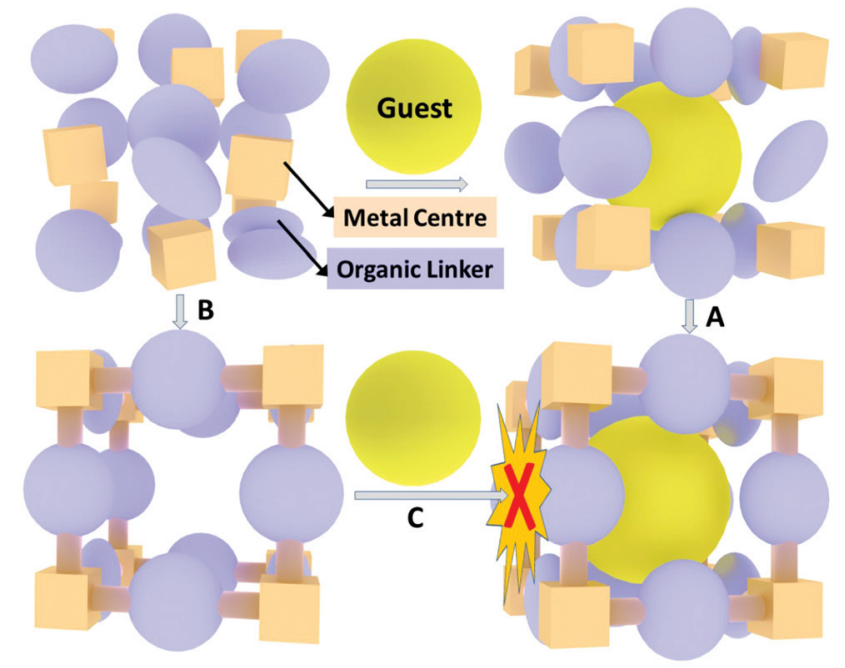

Scheme 1 Schematic diagram illustrating the concept of in situ confinement of a bulky guest in the nanocage of a MOF coordination space (A). Typically this nanoscale void space will become inaccessible to a bulkier molecule after the completion of the MOF self-assembly process $(B, C)$, because of steric hindrance imposed by narrow pore window apertures.

in this field of research. Firstly, conventional ex situ encapsulation methods usually require long processing times; guest infiltration or exchange processes can consume many hours ${ }^{32}$ and this could extend to several days. ${ }^{33,40}$ Secondly, effective infiltration of MOFs is further limited to guest molecules whose size is typically smaller than that of the pore window aperture of the host framework material, even after taking into account framework flexibility associated with lattice dynamics. ${ }^{41}$

To this end, identification of a suitable MOF structure with the propensity to self-organize spontaneously (via mixing of organic linker and metal ion solutions under ambient conditions) would be fundamental to accomplish in situ encapsulation of molecular guests inside its pores. Specifically, the advantage of facile MOF synthesis (e.g. ZIF-8 nanocrystals) ${ }^{42}$ can be exploited to offer a straightforward route to confine a guest molecule bigger than the physical dimensions permitted by the MOF window opening. Taking into account the foregoing challenges, herein we demonstrate the feasibility of a rapid in situ nanoconfinement methodology (Scheme 1), for the first time, to achieve encapsulation of a bulky luminescent guest molecule in conjunction with (MOF) host self-assembly to yield photonic hybrid crystals.

\section{Results and discussion}

Host $\subset$ guest nanoconfinement strategy via hybrid selfassembly

In this study, we selected the zeolitic imidazolate framework-8 $(\mathrm{ZIF}-8)^{43}$ material to act as the nanoporous host structure, within which we encapsulate a luminescent molecular guest.
This strategy was motivated by the larger internal void size of ZIF-8 at $13.32 \AA \times 11.18 \AA$ (excluding van der Waals surface) viewed along its cubic cell axis (see Fig. 1(a) and (b)); noting that albeit its pore window aperture is much narrower with a diameter of $\sim 3.4$ A. While ZIF-8 is a topical MOF material whose sodalite topology ${ }^{44}$ is identical to that of inorganic zeolites, the former framework exhibits an appreciably lower elastic modulus ${ }^{45,46}$ (of at least a factor of ten times lower). ${ }^{47,48}$ Its intrinsically smaller window aperture may, however, hinder the true advantage of having a larger internal void space. Indeed recent studies showed that only small gas molecules could penetrate the internal void cavity of ZIF-8 upon application of a threshold pressure ${ }^{49}$ and as facilitated by flexible gate-opening dynamics. ${ }^{41}$

We applied the ease and rapidity of ZIF-8 nanocrystal formation (via simple mixing of 2-methylimidazole (mIm) and $\mathrm{Zn}\left(\mathrm{NO}_{3}\right)_{2}$ solutions in methanol $)^{50}$ in combination with the concept of in situ guest confinement (Scheme 1), to construct a new host-guest MOF hybrid composite (Fig. 1(c)) with tunable guest-induced electronic and optical emission properties. Herein the choice of a compatible guest molecule containing interaction sites for effective intermolecular interactions with the host framework is key. By considering the structural dimensions of ZIF-8 (Fig. 1), we identified the highly luminescent monomeric inorganic metal complex: Zn-(bis-8hydroxyquinoline), termed $\mathrm{ZnQ},{ }^{51}$ for encapsulation within the internal pores of ZIF-8. Noteworthily, ZnQ is one of the relatively smaller and yet highly luminescent (quantum yield $36.6 \%)^{52}$ metal complexes amongst the renowned metal quinolate $\mathrm{Mq}_{n}$ series (e.g. $\left.\mathrm{Alq}_{3}, \mathrm{Gaq}_{3}, \mathrm{Inq}_{3}\right) .{ }^{53}$ Molecular dimensions of the ZnQ metal complex is approximately $11.74 \AA \times 6.49 \AA$ (with van der Waals radii), which is geometrically favorable for nanoconfinement within the pore of ZIF-8 (see Fig. S1 in the ESI $\dagger$ ). Despite many previous studies dealing with the application of luminescent $\mathrm{ZnQ}$ complexes alone ${ }^{53-55}$ to the best of our knowledge this paper reports the first demonstration of the host-guest emission effect contrived by in situ nanoconfinement of $\mathrm{ZnQ}$ within a porous optically inactive MOF. Our proposed methodology is unique compared to those found on guest-encapsulated luminescent MOFs. ${ }^{24,26,56}$

To obtain host-guest hybrid complexation of ZIF-8 $\supset$ ZnQ, we first attempted the material synthesis by the direct addition of $\mathrm{Zn}^{2+}$ cations into a transparent solution containing an equal amount of 2-methylimidazole $(\mathrm{mIm})$ and 8-hydroxyquinoline (8HQ) linkers. Nonetheless, we discovered that such an approach would only result in an instantaneous formation of ZnQ guests (confirmed by immediate intense yellow coloration of the solution), whereas the crystallization of ZIF-8 was suppressed completely. Accordingly it became evident that since we are dealing with a common metal ion in both the host and guest, the coordination tendency of the 8HQ linker towards $\mathrm{Zn}^{2+}$ is considerably stronger than the mIm linker, due to the presence of the electron-rich naphthalene moiety (constituting $\mathrm{O}, \mathrm{N}$ donors) in $8 \mathrm{HQ}$ compared to the 5 -membered mIm ring (comprising one secondary and one tertiary amine sites). To overcome this obstacle for accomplishing reactions (1) and (2), 
a)

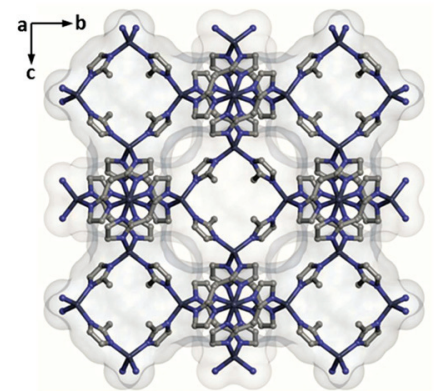

b)

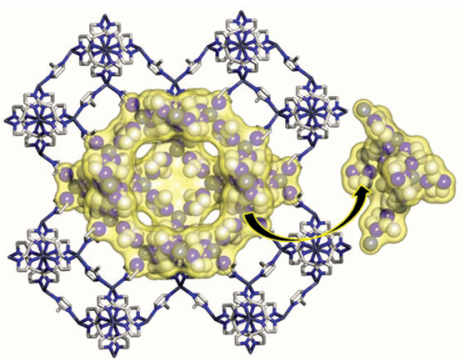

1

c)

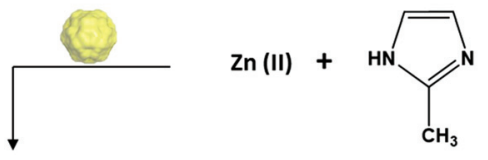

$\downarrow$

Fig. 1 (a) Packing diagram of a ZIF-8 cubic unit cell depicting the smaller aperture window openings leading towards (b) the larger internal void volume at the center of the sodalite nanocage. (c) Schematics depict in situ encapsulation of a bulkier guest molecule whose dimension greatly exceeds the window openings of ZIF-8.

Table 1 Solution-based chemical reactions designated as I to IV, conducted at $80{ }^{\circ} \mathrm{C}$ using different combinations of starting reactant concentrations (in $\mathrm{mmol}$ ). The detailed synthesis procedure is described in the Experimental methods section at the end. The products were characterized by X-ray, microscopy and spectroscopy techniques to determine their crystallinity and phases

\begin{tabular}{|c|c|c|c|c|c|}
\hline \multirow[b]{2}{*}{ Reaction no. } & \multicolumn{2}{|c|}{ ZnQ guest } & \multicolumn{2}{|c|}{ ZIF-8 host } & \multirow[b]{2}{*}{ Products } \\
\hline & $\mathrm{Zn}^{2+}$ & $8 \mathrm{HQ}$ & $\mathrm{Zn}^{2+}$ & $\mathrm{mIm}$ & \\
\hline $\mathbf{I}$ & 3 & 6 & 4 & 16 & Impure product mixtures \\
\hline II & 2 & 4 & 4 & 16 & Impure product mixtures \\
\hline III & 1 & 2 & 4 & 16 & No crystallization \\
\hline IV & 0.5 & 1 & 4 & 16 & Phase pure nanocrystals \\
\hline
\end{tabular}

we have designed a set of reactions by maintaining a constant ratio of $\mathrm{Zn}^{2+}$ : $\mathrm{mIm}$ (i.e. ZIF-8 formation) but systematically adjusting the concentrations of $\mathrm{Zn}^{2+}: 8 \mathrm{HQ}$ (i.e. $\mathrm{ZnQ}$ formation), in accordance with Table 1.

$$
\begin{gathered}
\mathrm{Zn}^{2+}+8 \mathrm{HQ}=\mathrm{ZnQ} \\
\mathrm{ZnQ}+\mathrm{Zn}^{2+}+\mathrm{mIm}=\mathrm{ZIF}-8 \supset \mathrm{ZnQ}
\end{gathered}
$$

Results summarizing different combinations of reactions (Table 1) provide important insights into the optimal concentration of ZnQ guest molecules required for successful confinement in the ZIF-8 host. For instance, the application of a high concentration of $\mathrm{ZnQ}$ in reactions I and II yielded non phasepure products containing a mixture of crystalline stable forms of ZnQ complexes (Fig. S2†). This outcome can be explained by the strong chelating effects of $8 \mathrm{HQ}$ forcing the oligomeriza- tion of monomers into tetramers. ${ }^{55}$ As a corollary, raising the concentration of ZnQ may encourage the formation of $\mathrm{ZnQ}$ stable species, instead of achieving guest encapsulation inside the MOF pores. In particular we found that reaction IV utilizing a much lower concentration of $\mathrm{ZnQ}$ complex produced a purely crystalline phase (Fig. S3†); in contrast reaction III formed no crystalline products, implying that $\mathrm{ZnQ}$ converts into $\left(\mathrm{ZnQ}_{2}\right)_{n}$ oligomers that remain stable in the solution state. We pinpointed reaction IV as the optimal route for performing successful ZIF-8 $\supset$ ZnQ encapsulation reaction, thereby the properties of this sample are studied in detail below.

\section{Morphological and structural characterization of hybrid crystals}

Visual inspections indicated that the pure crystalline dried product obtained from reaction IV (Table 1) showed a pale ivory color, unlike the distinctively white color of pristine ZIF-8, or the yellow color characteristic of pristine ZnQ powder. Optical and transmission electron microscopy (TEM) images of this sample confirmed a rhombic dodecahedral morphology (of twelve facets) ${ }^{42}$ and, its crystal size in the range of $500 \mathrm{~nm}$ to $2 \mu \mathrm{m}$ (see Fig. 2(a), (b) and S1 $\dagger$ ). Comprehensive information about the crystal size distribution was further determined by means of dynamic light scattering (DLS) experiments. It can be seen in Fig. 2(c) that the pristine ZIF-8 nanocrystals exist in the range of $200-500 \mathrm{~nm}$, as synthesized at room temperature using methanol. In contrast, we established from Fig. 2(d) that the nanoconfinement process to afford encapsulation of the ZnQ guest within ZIF-8 has resulted in a relatively larger crystal size distribution between 

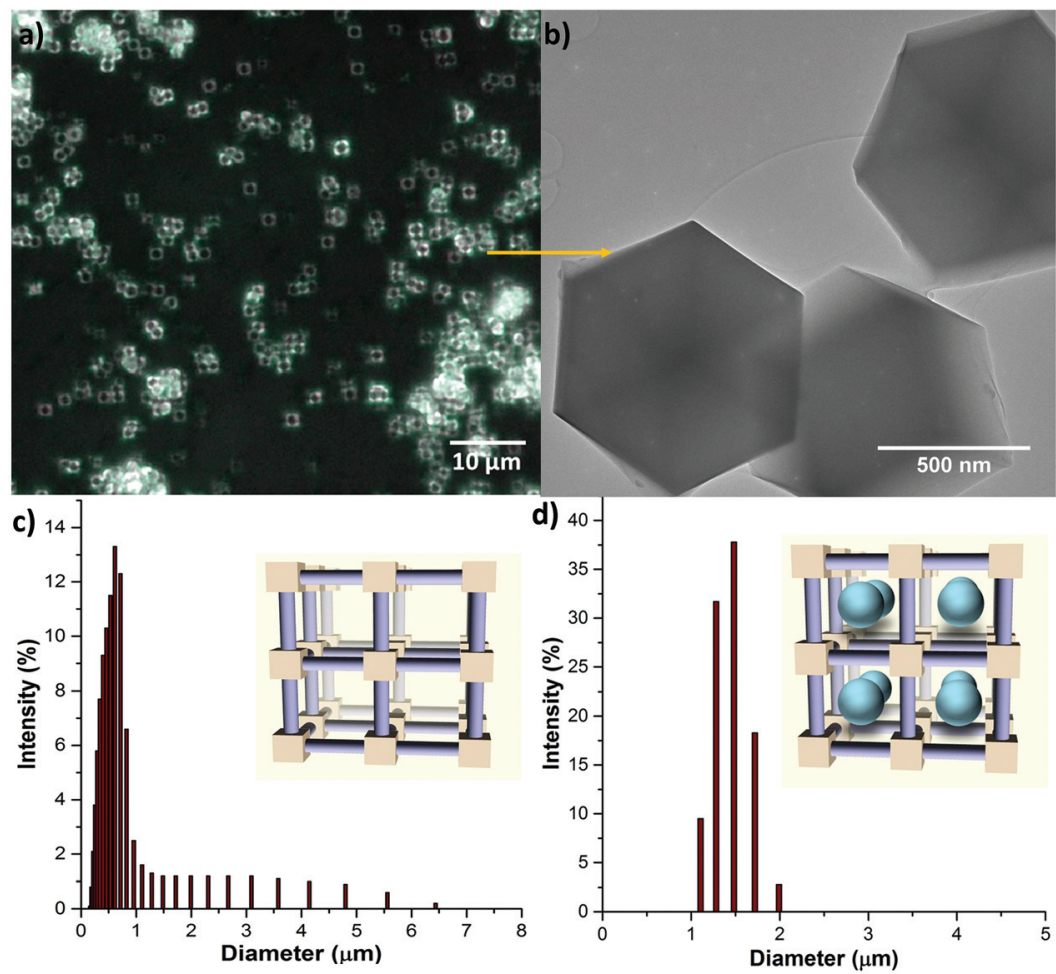

Fig. 2 (a) Optical micrograph of ZIF-8 $\supset$ ZnQ crystals and (b) the corresponding TEM image showing the single-crystal rhombic dodecahedral morphology. Dynamic light scattering spectra of (c) pristine ZIF-8 versus (d) encapsulated ZIF-8 $\supset$ ZnQ.

$800 \mathrm{~nm}$ and $2 \mu \mathrm{m}$. The DLS data suggest that the co-operative self-assembly route (Fig. 1) responsible for guest species encapsulation could also stimulate the growth rate of ZIF-8 nanocrystals.

Fig. 3 presents the structural data of the pristine ZIF-8 material compared with its guest-encapsulated counterpart. Powder X-ray diffraction data (PXRD) in Fig. 3(a) show the structural integrity of ZIF-8 $\supset$ ZnQ evidenced from the sharp peaks and the phase purity of the bulk sample, showing a good agreement with the simulated X-ray pattern of ZIF-8. Likewise, vibrational data measured by Raman and infrared (IR) spectroscopy further demonstrated matching signatures in agreement with the pristine ZIF-8 compound (Fig. 3(b) and (c)). Together we found that the ZIF-8 host has retained its pristine structural form, upon confinement of the ZnQ guests, for which no major structural changes have been observed. This behavior is similar to examples in the literature which reported no change in the structural X-ray diffraction pattern upon guest encapsulation in the ZIF- ${ }^{36}$ and ZIF- $900^{56}$ host compounds.

However, thermogravimetric analysis (TGA) of hybrid and pure materials (Fig. 4) has enabled us to unambiguously confirm not only guest confinement in ZIF-8 pores, but also revealing enhanced thermal stability of ZnQ guests upon confinement. It can be seen in Fig. 4 that the (pure) ZIF-8 host exhibited thermal stability until $\sim 280{ }^{\circ} \mathrm{C}$, however it showed a two-step weight loss profile for ZIF-8 $\supset$ ZnQ host-guest hybrid crystals. First, the distinctive $4 \%$ weight loss from
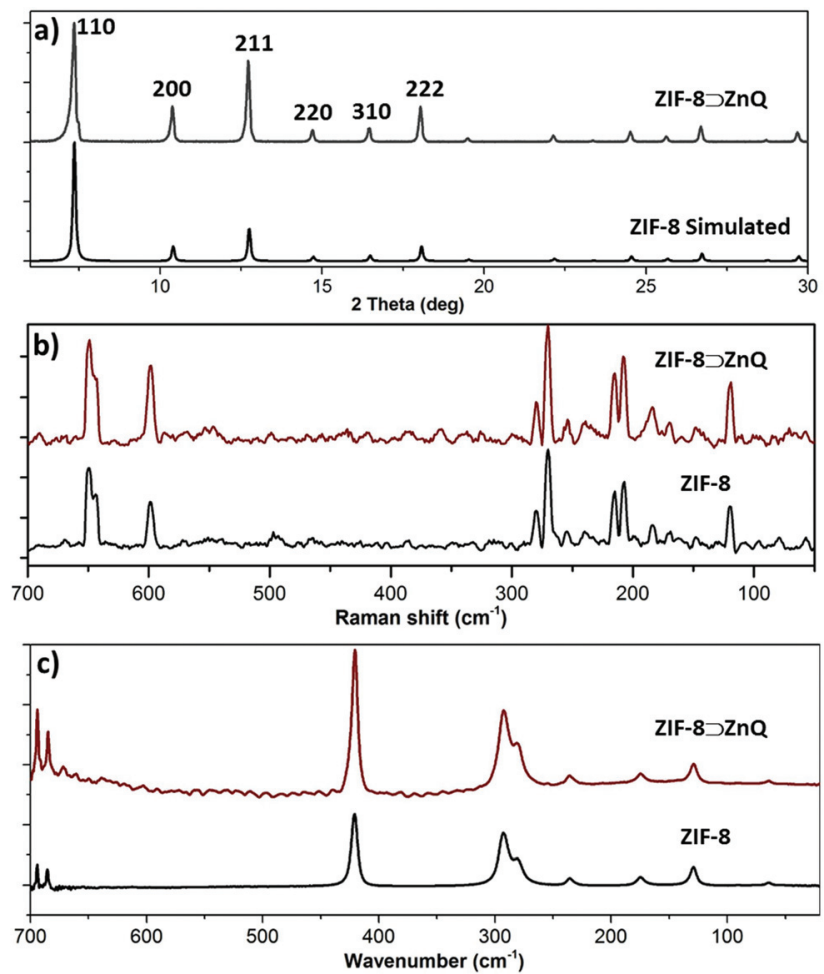

Fig. 3 Structural characterization of encapsulated ZIF-8 $\supset$ ZnQ and pristine ZIF-8. (a) Powder X-ray diffraction pattern, (b) Raman vibrational spectra, and (c) infrared spectra. Note that the vertical axis represents intensity in arbitrary units. 
$200{ }^{\circ} \mathrm{C}$ to $235{ }^{\circ} \mathrm{C}$ could be ascribed to the coordinated water molecules in ZnQ guests; second, slow decomposition from $235{ }^{\circ} \mathrm{C}$ to $410{ }^{\circ} \mathrm{C}$ corresponds to the integrated structural decomposition of the ZIF-8 host and its encapsulated guest; and third, rapid chemical decomposition dominates beyond $\sim 410{ }^{\circ} \mathrm{C}$. Notably our TGA data suggest that due to the nanoconfinement effect both the coordinated water molecules (loss at $180{ }^{\circ} \mathrm{C}$ for pure $\mathrm{ZnQ}$, but increased to $200{ }^{\circ} \mathrm{C}$ for confined form) and the remaining guest molecule (slow decomposition from $200{ }^{\circ} \mathrm{C}$ for pure $\mathrm{ZnQ}$ but increased to $235^{\circ} \mathrm{C}$ for confined form) are decomposing at relatively higher temperatures compared to their non-encapsulated (pure) forms.

\section{Optical spectra and electronic transitions phenomena}

Absorption and emission optical spectra of the ZIF-8 host, ZnQ guest, and encapsulated ZIF-8 $\supset$ ZnQ hybrid are presented in Fig. 5, which allow us to elucidate the underlying host-guest interaction behaviors. Specifically, it can be seen in Fig. 5(a) that pristine ZIF-8 exhibits a strong absorption peak at $221 \mathrm{~nm}$, alongside two weaker absorption peaks at $304 \mathrm{~nm}$ and $370 \mathrm{~nm}$ (inset). On the other hand, the pure ZnQ metal complex has a broad absorption peak at $412 \mathrm{~nm}$ accompanied by a small distinctive hump at $261 \mathrm{~nm}$ (inset), which can be assigned to the intermolecular $\pi-\pi$ interaction and the $\pi-\pi^{*}$ transition events, respectively.

It is important to recognize that the characteristic absorption spectrum for the hybrid material, i.e. host-guest ZIF-8 ZnQ in Fig. 5(a), exhibits distinguishable identities of both the host and the guest species though with apparent deviations in peak profiles. More precisely, we established three main characteristics: (i) a sharp peak of ZIF-8 at $221 \mathrm{~nm}$ was detected at the original position, but with an additional small hump at the base (266 nm) corresponding to the ZnQ guest. (ii) Absorption peaks reminiscent of $\mathrm{ZnQ}$ were recorded in the host-guest hybrid, but of a narrower peak area compared to the broader bands found in pristine ZnQ. (iii) Distinct shifts in peak positions were identified for $\mathrm{ZnQ}$ after guest encapsulation. Specifically, the red-shifted hump at $335 \mathrm{~nm}$ could be assigned to the stabilization of the excited state $\pi-\pi^{*}$ transition, while the blue-shifted main absorption peak at $384 \mathrm{~nm}$
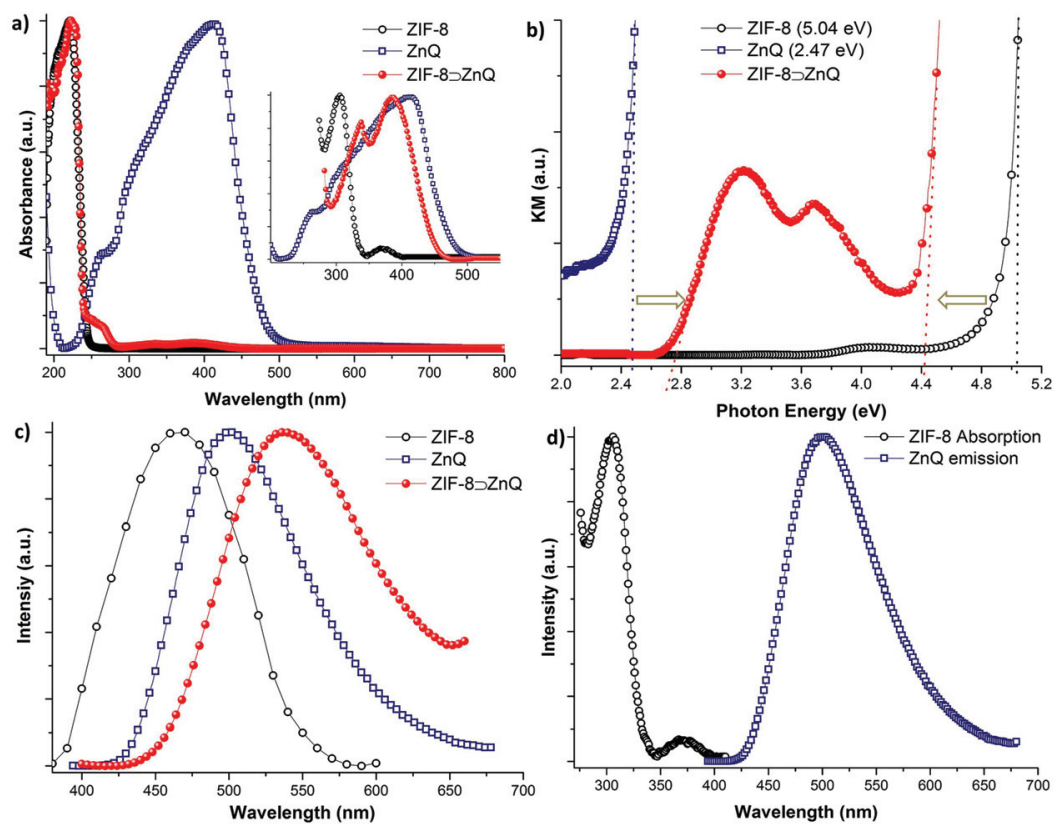

Fig. 5 (a) UV-Vis absorption spectra of the host and guest versus that of the encapsulated compound; the inset shows an enlarged view of the weak absorption region. (b) Kubelka-Munk (KM) function to determine band gap values at the designated photon energy intercepts. (c) Fluorescence emission spectra of the three compounds excited at $350 \mathrm{~nm}$. (d) Emission and absorption spectra of ZnQ and ZIF-8, respectively. 


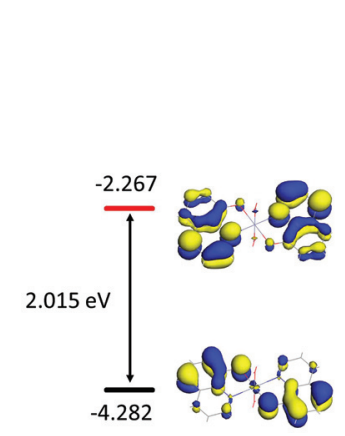

15 carbon; white: hydrogen].
$\mathrm{znQ}$

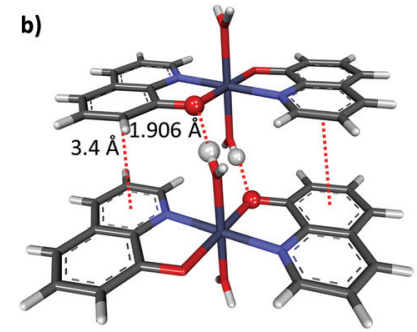

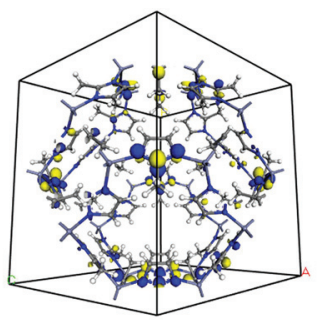

$5.470 \mathrm{eV}$

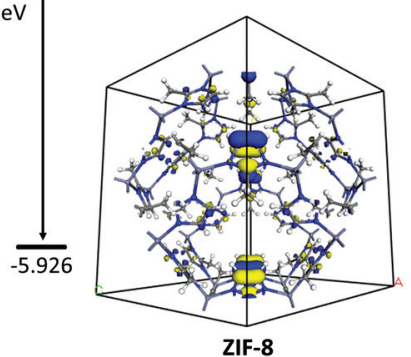

c)

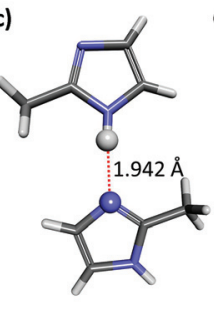

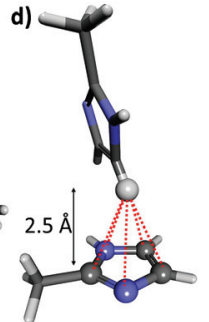

Fig. 6 (a) HOMO-LUMO energy levels for ZnQ and ZIF-8 and their corresponding band gaps computed from DFT. (b) Weak intermolecular interactions in $\mathrm{ZnQ}$ crystal packing showing $\pi \cdots \pi$ and $\mathrm{O} \cdots \mathrm{H}$ interactions. $^{60}$ (c) Hydrogen bonding and (d) $\mathrm{N}-\mathrm{H} \cdots \pi$ interactions of the $\mathrm{mlm}$ packing. ${ }^{61}$ [Red: oxygen; dark blue: zinc; light blue: nitrogen; gray:

is attributed to the spatial confinement (i.e. caging effect) ${ }^{57}$ of the guest molecule imposed by the sodalite cage of ZIF-8.

We have measured the change in band gap values of the ZIF-8 $\supset$ ZnQ hybrid material compared to the pristine ZIF-8 or ZnQ by adopting the Kubelka-Munk (KM) function, whose results are shown in Fig. 5(b). The band gap of pristine ZIF-8 $\left(E_{\mathrm{g}}=5.04 \mathrm{eV}\right.$ ) was found to decrease by $0.63 \mathrm{eV}$ (to $E_{\mathrm{g}}=$ $4.41 \mathrm{eV}$ ) once the $\mathrm{ZnQ}$ guest has been encapsulated to form ZIF-8 $\supset$ ZnQ. On the contrary, encapsulated ZnQ shows a band gap of $2.74 \mathrm{eV}$, which is $0.27 \mathrm{eV}$ higher than the isolated $\mathrm{ZnQ}$ complex $\left(E_{\mathrm{g}}=2.47 \mathrm{eV}\right)$. Additionally, we performed theoretical orbital energy calculations that predicted band gap values of $5.47 \mathrm{eV}$ for ZIF-8 and 2.02 eV for ZnQ (Fig. 6(a)); details of the density functional calculations are given in the ESI. $\dagger$ It is worth highlighting that the experimental band gap of ZIF-8 as well as the theoretically calculated values obtained in this work are consistent with the experimental ${ }^{58}$ and calculated ${ }^{59}$ magnitudes from the recent literature. Fluorescence spectra in Fig. 5(c) provide evidence of co-operative host-guest intermolecular interactions responsible for the red-shifted emission spectrum evidenced in ZIF-8 $\supset$ ZnQ. It can be seen that the maximum emission peak of $\mathrm{ZnQ}$ at $\sim 500 \mathrm{~nm}$ has red- shifted by $\sim 36 \mathrm{~nm}$ to a higher wavelength of $\lambda_{\mathrm{em}} \sim 536 \mathrm{~nm}$, which occurred upon encapsulation of ZnQ guests inside the sodalite cage of ZIF-8 (pristine $\lambda_{\mathrm{em}} \sim 466 \mathrm{~nm}$ ). To understand the possible excitation mechanism behind such a host-guest interaction, we will consider donor-acceptor type emissionabsorption spectra (Fig. 5(d)), and also examine the HOMOLUMO theoretical orbital energies of ZIF-8 against that of ZnQ (Fig. 6(a)). Interestingly, from the absorption spectrum of ZIF-8 (the anticipated acceptor) compared with the emission band of $\mathrm{ZnQ}$ (the anticipated electron-rich donor), we found no overlap between the isolated host and guest (see Fig. 5(d)), suggesting that there is no energy transfer from ZnQ to ZIF-8. On the basis of the HOMO and LUMO orbital alignments predicted by DFT calculations (Fig. 6(a)), we postulated that the higher-energy LUMO orbital of ZIF-8 could transfer its charge (harvested upon excitation) onto the low-lying LUMO orbital of ZnQ, through an intermolecular weak interaction established by guest confinement. This energy transfer mechanism can shift the emission band to much higher wavelengths as witnessed in our experiments (Fig. 5(c)).

To further corroborate the photophysical phenomena presented above, we now examine the detailed crystal structures of the mIm linker (2-methylimidazole) and the ZnQ complex from the perspective of their propensity to develop intermolecular bonding interactions. Fig. 6(b) depicts the crystalpacking diagram of the ZnQ metal complex, ${ }^{60}$ showing the parallel offset type $\pi \cdots \pi$ stacking interaction with a neighboring ZnQ molecule (whose spacing, $d=3.4 \AA$ ), together with the presence of strong intermolecular hydrogen bonding $(d=$ $1.906 \AA)$ from its coordinated water molecules to the other 'O'-site (of 8HQ) coordinated to the Zn center of an adjacent ZnQ molecule. Turning to the mIm organic molecule, Fig. 6(c) and (d) show the reported crystal configurations ${ }^{61}$ revealing its ability to form strong $\mathrm{N}-\mathrm{H} \cdots \pi$ interactions with an adjacent mIm molecule $(d=2.5 \AA)$, plus an intramolecular $\mathrm{N} \cdots \mathrm{H}$ bonding $(d=1.942 \AA)$. Hence in the context of the ZIF-8 $\supset$ ZnQ hybrid, its spatially confined host-guest intermolecular environment consists of: (i) $\pi \cdots \pi$ interactions established between the electron-rich aromatic moiety of the $\mathrm{ZnQ}$ guest and the mIm linkages of the ZIF-8 host, in conjunction with (ii) hydrogen bonding associated with the coordinated water molecules in $\mathrm{ZnQ}$ guests to the $\mathrm{N}$ sites situated on the $\mathrm{ZnN}_{4}$ tetrahedra of the sodalite ZIF-8 host.

\section{Emission color chromaticity and photophysical stability}

We have fabricated simple proof-of-principle LED-based lighting devices (Fig. 7(a) and (b)) featuring a thin functional coating of active material made from ZIF-8 $\supset$ ZnQ. The improvement in luminescence intensity attributed to guest encapsulation was visibly detectable under the excitation of an ultraviolet (UV) LED source. Significantly, it can be seen in Fig. 7(a) that the ZIF-8 $\supset$ ZnQ coating emits light-yellow emission under UV excitation, whereas the pristine ZIF-8 coating in Fig. 7(b) is essentially inactive (note that blue light is transmitted from the UV source). Furthermore, we have evaluated the CIE chromaticity color coordinates shown in Fig. 7(c), and 

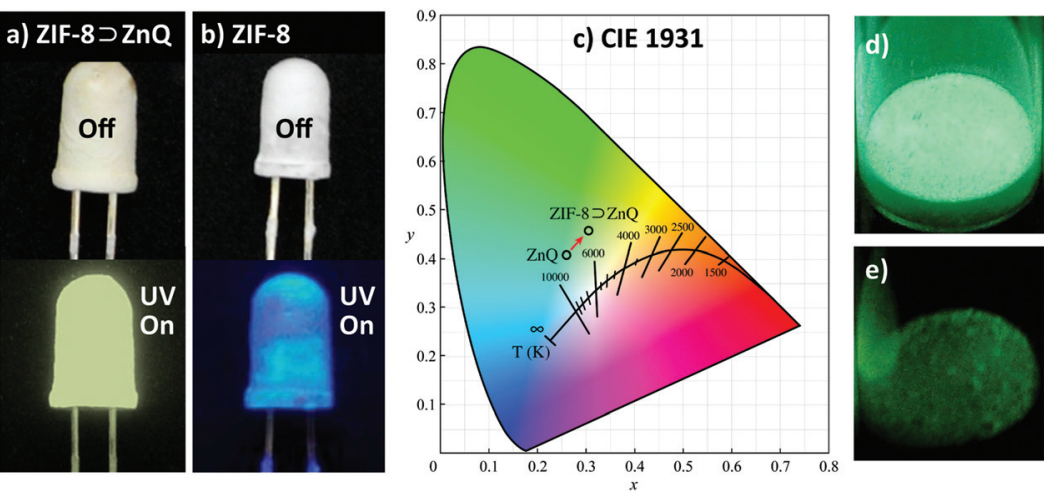

Fig. 7 Visible light photographs showing UV LED (switched off) coated with a thin layer of (a) encapsulated ZIF-8 $\supset$ ZnQ versus (b) pristine ZIF-8; the bottom panel shows photographs of the corresponding devices taken in the dark with the UV LED switched on, showing ZIF- $8 \supset$ ZnQ with light yellow emission and inactive ZIF-8 (blue intensity is from UV source). (c) CIE 1931 color coordinates space of pure ZnQ ( $x$ : 0.2567, $y$ : 0.4096) compared with the encapsulated ZIF-8 $\supset$ ZnQ (x: 0.3055, y: 0.4630). (d) Emission of a freshly synthesized dry powder sample of ZnQ under exposure to UV light, and (e) its diminished luminescence after exposure to sunlight for four days.

determined the correlated color temperature (CCT) of the samples. It is worth highlighting that the CCT of the pristine $\mathrm{ZnQ}$ is at $\sim 8188 \mathrm{~K}$ (i.e. amongst cool colors), but this reduces to $\sim 6196 \mathrm{~K}$ for ZIF-8 $\supset \mathrm{ZnQ}$ after nanoconfinement of the $\mathrm{ZnQ}$ guest in the sodalite cage of ZIF-8, which translates into a perceptible red-shift towards a relatively warmer color rendering (Fig. 7(c)).

Another important observation concerns the preservation of the luminescence properties of the encapsulated-guest species by nanoconfinement in the MOF host. Surprisingly, we discovered that the ZIF-8 $\supset$ ZnQ hybrid exhibits enhanced photostability, see Fig. 7(d) and (e), whereas the 'unprotected' ZnQ complex is highly susceptible to photochemical degradation upon exposure to sunlight. Remarkably, we witnessed that the photostability of the ZnQ emitter when shielded by the ZIF- 8 pores is at least twice more stable than the unprotected ZnQ guests (see Fig. S5, $\uparrow$ showing relative photostability data for a period of 5 days). Moreover, from the Raman experiments we observed that a laser power of $50 \mathrm{~mW}$ was adequate to instantaneously damage the ZnQ sample causing complete photochemical decomposition (Fig. S4†), however the encapsulated sample remained stable when tested under the same conditions. This provides additional evidence demonstrating the poor photostability of $\mathrm{ZnQ}$ against monochromatic laser light (e.g. $532 \mathrm{~nm}, 12.5$ to $50 \mathrm{~mW}$ ), whereas we found the encapsulated $\mathrm{ZnQ}$ to retain its optical properties after nanoconfinement in the ZIF-8 pore. Our experimental observations support the notion that the trapping of light-sensitive guest molecules by caging them in the ordered porous architecture of MOFs may offer a simple method of shielding against swift photoinduced decay, or for precisely controlling photochemical processes at the desired rates.

\section{Conclusions}

We have demonstrated the efficacy of the concept of rapid in situ host-guest nanoconfinement, where encapsulation of a highly-emitting ZnQ guest molecule in the long-range ordered porosity of ZIF-8 yielded a hybrid nanomaterial with bespoke optoelectronic properties and enhanced photostability. One attraction of the proposed approach lies in its capacity to confine bulky guest molecules in certain MOF hosts, particularly the ones featuring narrow window apertures that inevitably prevent conventional (ex situ) guest infiltration or ionexchange strategies. In this study, we discovered that there is a strong co-operative intermolecular interaction of the guest species with the framework host material, giving rise to a distinctive red-shifted emission measured in the ZIF-8 $\supset$ ZnQ hybrid crystals. We gathered evidence indicating that the sodalite cage of ZIF-8 not only provides stabilization of the guest via the spatial nanoconfinement mechanism, but the host may also function as a photon harvester transferring charge to the encapsulated guest via intimate host-guest electronic pathways. We demonstrate that enhancement in photochemical stability can be accomplished through host-guest nanoconfinement within the molecular vessels of ZIF-8. It is envisioned that this strategy can be extended to nano-caging and nanomanipulation of a wide variety of light-sensitive fluorophores combined with the diverse family of MOF hosts ${ }^{62,63}$ and porous structures ${ }^{64}$ that are found in the literature. Indeed improving the photostability performance and overall longterm material durability through nanoporous host-guest encapsulation is important in the field of light-matter interactions science, with many exemplars already demonstrated for inorganic zeolites, ${ }^{65}$ self-assembled cavitand-based capsules, ${ }^{66}$ and molecular sieves $;{ }^{67}$ the current study offers an attractive strategy whereby MOF can also be utilized to achieve such an effect.

\section{Experimental methods}

\section{In situ synthesis of the ZIF-8 $\supset$ ZnQ host-guest system}

The hybrid host-guest material was synthesized employing the combination reaction IV in Table 1, where ZnQ was obtained 
from a concentration ratio of $0.5: 1 \mathrm{mmol}\left(\mathrm{Zn}^{2+}: 8 \mathrm{HQ}\right)$. Initially, $\mathrm{ZnQ}$ was synthesized by mixing $\mathrm{Zn}^{2+}$ and $8 \mathrm{HQ}$ in $25 \mathrm{~mL}$ of methanol. In another flask, $4: 16 \mathrm{mmol}$ of $\mathrm{Zn}^{2+}$ : mIm was added to $25 \mathrm{~mL}$ of methanol. This solution was kept stirred for 5 minutes and after that the ZnQ solution was added slowly. After vigorous stirring, the temperature was increased to $80{ }^{\circ} \mathrm{C}$. Yellow colloidal nanocrystals started to appear at this stage and we found that the product yield can be further maximized by adding an excess amount of both $\mathrm{Zn}^{2+}$ and mIm in a 1:4 mmol ratio. The synthesis took about 10 minutes. The crystalline product was washed thoroughly (4 cycles) using a copious amount of methanol and acetone to remove any excess ZnQ compound and residual soluble products. The final product was separated by centrifugation at $8000 \mathrm{rpm}$.

\section{Materials characterization techniques}

All compounds were characterized at room temperature on dried powder samples using a combination of X-ray, spectroscopy and microscopy techniques. Powder X-ray diffraction (PXRD) was performed using a Rigaku Smart Lab diffractometer with a $\mathrm{Cu} \mathrm{K} \alpha$ source $(1.541 \AA)$, where diffraction data were collected at $2 \theta$ angle from $2^{\circ}$ to $30^{\circ}$, using a $0.01^{\circ}$ step size and $1^{\circ} \mathrm{min}^{-1}$ step speed. Raman spectroscopy was conducted on a Bruker Senterra dispersive Raman microscope equipped with $532 \mathrm{~nm}, 633 \mathrm{~nm}$ and $785 \mathrm{~nm}$ lasers; infrared absorption spectroscopy was performed on a Bruker Vertex 80 V FTIR interferometer. Solid state diffuse reflectance spectra were recorded on a UV-2600 Shimadzu spectrophotometer using a standard sample holder. Solid state emission was measured using the Horiba FluoroMax spectrofluorometer at a fixed slit width of $5 \mathrm{~nm}$ and a constant excitation wavelength of $350 \mathrm{~nm}$. The CIE chromaticity was determined utilizing the UPRtek MK350N+ spectrometer. The optical images of the nanocrystals were captured using the Alicona InfiniteFocus 3D microscope, while TEM images were acquired using the JEOL JEM-2100 LaB6 transmission electron microscope at $200 \mathrm{kV}$. Dynamic light scattering measurements were carried out using the Malvern Zetasizer Nano ZS ZEN3600.

\section{$45 \quad$ Acknowledgements}

This research is supported by Samsung Advanced Institute of Technology (SAIT GRO DFR00230) and The Royal Society Research Grant (RG140296). We thank the Research Complex at Harwell (RCaH), Oxfordshire, for access to the advanced materials characterization suite. We acknowledge the ISIS Rutherford Appleton Laboratory, particularly Dr Jeff Sykora (R63 ISIS Instrumentation Division), Dr Marek Jura and Dr Gavin Stenning (R53 Materials Characterization Lab), and Dr James Taylor (R79 Hydrogen and Catalysis Lab) for the provision of spectrometers and X-ray diffractometer. We are grateful to Dr Gianfelice Cinque at Diamond Light Source (B22 MIRIAM) for access to the Raman and FTIR spectrometers.

\section{References}

1 R. H. Friend, R. W. Gymer, A. B. Holmes, J. H. Burroughes, R. N. Marks, C. Taliani, D. D. C. Bradley, D. A. Dos Santos, J. L. Bredas, M. Logdlund and W. R. Salaneck, Nature, 1999, 397, 121-128.

2 S. Reineke, M. Thomschke, B. Lüssem and K. Leo, Rev. Mod. Phys., 2013, 85, 1245-1293.

3 A. Cadiau, C. D. Brites, P. M. Costa, R. A. Ferreira, J. Rocha and L. D. Carlos, ACS Nano, 2013, 7, 7213-7218.

4 L. Prodi, F. Bolletta, M. Montalti and N. Zaccheroni, Coord. Chem. Rev., 2000, 205, 59-83.

5 Y. Zhou and B. Yan, Nanoscale, 2015, 7, 4063-4069.

6 A. P. de Silva, H. Q. Gunaratne, T. Gunnlaugsson, A. J. Huxley, C. P. McCoy, J. T. Rademacher and T. E. Rice, Chem. Rev., 1997, 97, 1515-1566.

7 L. Heinke, M. Cakici, M. Dommaschk, S. Grosjean, R. Herges, S. Brase and C. Woll, ACS Nano, 2014, 8, 14631467.

8 Y. Yang, Q. Zhao, W. Feng and F. Li, Chem. Rev., 2013, 113, 192-270.

9 S. R. Forrest, Nature, 2004, 428, 911-918.

10 S. V. Eliseeva and J. C. Bunzli, Chem. Soc. Rev., 2010, 39, 189-227.

11 K. F. Mak, C. Lee, J. Hone, J. Shan and T. F. Heinz, Phys. Rev. Lett., 2010, 105.

12 L. Pavesi, L. Dal Negro, C. Mazzoleni, G. Franzo and F. Priolo, Nature, 2000, 408, 440-444.

13 C. N. R. Rao, A. K. Cheetham and A. Thirumurugan, J. Phys.: Condens. Matter, 2008, 20, 083202.

14 O. Bisi, S. Ossicini and L. Pavesi, Surf. Sci. Rep., 2000, 38, 1-126.

15 P. V. Kamat, J. Phys. Chem. C, 2007, 111, 2834-2860.

16 B. F. Hoskins and R. Robson, J. Am. Chem. Soc., 1989, 111, 5962-5964.

17 S. Kitagawa, R. Kitaura and S. Noro, Angew. Chem., Int. Ed., 2004, 43, 2334-2375.

18 H. C. Zhou, J. R. Long and O. M. Yaghi, Chem. Rev., 2012, 112, 673-674.

19 M. R. Ryder and J. C. Tan, Mater. Sci. Technol., 2014, 30, 1598-1612.

20 V. Stavila, A. A. Talin and M. D. Allendorf, Chem. Soc. Rev., 2014, 43, 5994-6010.

21 J. H. Lee, J. Jaworski and J. H. Jung, Nanoscale, 2013, 5, 8533-8540.

22 S. Horike, S. Shimomura and S. Kitagawa, Nat. Chem., 2009, 1, 695-704.

23 A. K. Chaudhari, I. Han and J. C. Tan, Adv. Mater., 2015, 27, 4438-4446.

24 M. D. Allendorf, M. E. Foster, F. Leonard, V. Stavila, P. L. Feng, F. P. Doty, K. Leong, E. Y. Ma, S. R. Johnston and A. A. Talin, J. Phys. Chem. Lett., 2015, 6, 1182-1195.

25 Y. Cui, Y. Yue, G. Qian and B. Chen, Chem. Rev., 2012, 112, 1126-1162.

26 Z. Hu, B. J. Deibert and J. Li, Chem. Soc. Rev., 2014, 43, 5815-5840. 
27 L. E. Kreno, K. Leong, O. K. Farha, M. Allendorf, R. P. Van Duyne and J. T. Hupp, Chem. Rev., 2012, 112, 1105-1125.

28 H. Khajavi, J. Gascon, J. M. Schins, L. D. A. Siebbeles and F. Kapteijn, J. Phys. Chem. C, 2011, 115, 12487-12493.

29 C. Wang, Z. Xie, K. E. deKrafft and W. Lin, J. Am. Chem. Soc., 2011, 133, 13445-13454.

30 W. A. Maza, R. Padilla and A. J. Morris, J. Am. Chem. Soc., 2015, 137, 8161-8168.

31 C. Y. Sun, X. L. Wang, X. Zhang, C. Qin, P. Li, Z. M. Su, D. X. Zhu, G. G. Shan, K. Z. Shao, H. Wu and J. Li, Nat. Commun., 2013, 4, 2717.

32 Y. Cui, T. Song, J. Yu, Y. Yang, Z. Wang and G. Qian, Adv. Funct. Mater., 2015, 25, 4796-4802.

33 J. Yu, Y. Cui, H. Xu, Y. Yang, Z. Wang, B. Chen and G. Qian, Nat. Commun., 2013, 4, 2719.

34 O. Shekhah, A. Cadiau and M. Eddaoudi, CrystEngComm, 2014, DOI: 10.1039/c4ce01402b.

35 D. Buso, J. Jasieniak, M. D. Lay, P. Schiavuta, P. Scopece, J. Laird, H. Amenitsch, A. J. Hill and P. Falcaro, Small, 2012, 8, 80-88.

36 B. P. Biswal, D. B. Shinde, V. K. Pillai and R. Banerjee, Nanoscale, 2013, 5, 10556-10561.

37 G. Lu, S. Li, Z. Guo, O. K. Farha, B. G. Hauser, X. Qi, Y. Wang, X. Wang, S. Han, X. Liu, J. S. DuChene, H. Zhang, Q. Zhang, X. Chen, J. Ma, S. C. Loo, W. D. Wei, Y. Yang, J. T. Hupp and F. Huo, Nat. Chem., 2012, 4, 310-316.

38 L. Shen, W. Wu, R. Liang, R. Lin and L. Wu, Nanoscale, 2013, 5, 9374-9382.

39 J. Zhuang, C. H. Kuo, L. Y. Chou, D. Y. Liu, E. Weerapana and C. K. Tsung, ACS Nano, 2014, 8, 2812-2819.

40 J. An and N. L. Rosi, J. Am. Chem. Soc., 2010, 132, 55785579.

41 M. R. Ryder, B. Civalleri, T. D. Bennett, S. Henke, S. Rudić, G. Cinque, F. Fernandez-Alonso and J. C. Tan, Phys. Rev. Lett., 2014, 113, 215502.

42 J. Cravillon, R. Nayuk, S. Springer, A. Feldhoff, K. Huber and M. Wiebcke, Chem. Mater., 2011, 23, 2130-2141.

43 K. S. Park, Z. Ni, A. P. Cote, J. Y. Choi, R. D. Huang, F. J. Uribe-Romo, H. K. Chae, M. O'Keeffe and O. M. Yaghi, Proc. Natl. Acad. Sci. U. S. A., 2006, 103, 10186-10191.

44 X. C. Huang, Y. Y. Lin, J. P. Zhang and X. M. Chen, Angew. Chem., Int. Ed., 2006, 45, 1557-1559.

45 J. C. Tan, B. Civalleri, C. C. Lin, L. Valenzano, R. Galvelis, P. F. Chen, T. D. Bennett, C. Mellot-Draznieks, C. M. Zicovich-Wilson and A. K. Cheetham, Phys. Rev. Lett., 2012, 108, 095502.

46 J. C. Tan, T. D. Bennett and A. K. Cheetham, Proc. Natl. Acad. Sci. U. S. A., 2010, 107, 9938-9943.
47 J. C. Tan, B. Civalleri, A. Erba and E. Albanese, CrystEngComm, 2015, 17, 375-382.

48 M. R. Ryder and J. C. Tan, Dalton Trans., 2016, DOI: 10.1039/C5DT03514G, (in press).

49 D. Fairen-Jimenez, S. A. Moggach, M. T. Wharmby, P. A. Wright, S. Parsons and T. Duren, J. Am. Chem. Soc., 2011, 133, 8900-8902.

50 Y. Pan, Y. Liu, G. Zeng, L. Zhao and Z. Lai, Chem. Commun., 2011, 47, 2071-2073.

51 Z.-F. Chen, M. Zhang, S.-M. Shi, L. Huang, H. Liang and Z.-Y. Zhou, Acta Crystallogr., Sect. E: Struct. Rep. Online, 2003, 59, 814-815.

52 R. F. Wang, Y. L. Cao, D. Z. Jia, L. Liu and F. Li, Opt. Mater., 2013, 36, 232-237.

53 P. E. Burrows, L. S. Sapochak, D. M. McCarty, S. R. Forrest and M. E. Thompson, Appl. Phys. Lett., 1994, 64, 27182720.

54 H. C. Pan, F. P. Liang, C. J. Mao, J. J. Zhu and H. Y. Chen, J. Phys. Chem. B, 2007, 111, 5767-5772.

55 L. S. Sapochak, F. E. Benincasa, R. S. Schofield, J. L. Baker, K. K. C. Riccio, D. Fogarty, H. Kohlmann, K. F. Ferris and P. E. Burrows, J. Am. Chem. Soc., 2002, 124, 6119-6125.

56 J. V. Morabito, L. Y. Chou, Z. Li, C. M. Manna, C. A. Petroff, R. J. Kyada, J. M. Palomba, J. A. Byers and C. K. Tsung, J. Am. Chem. Soc., 2014, 136, 12540-12543.

57 O. Shameema, C. N. Ramachandran and N. Sathyamurthy, J. Phys. Chem. A, 2006, 110, 2-4.

58 F. Wang, Z. S. Liu, H. Yang, Y. X. Tan and J. Zhang, Angew. Chem., Int. Ed., 2011, 50, 450-453.

59 K. T. Butler, C. H. Hendon and A. Walsh, ACS Appl. Mater. Interfaces, 2014, 6, 22044-22050.

60 Y. Q. Sun, Y. L. Lei, J. Gao, X. H. Sun, S. H. Lin, Q. L. Bao, Q. Liao, S. T. Lee and L. S. Liao, Chem. Commun., 2014, 50, 10812-10814.

61 B. Hachula, M. Nowak and J. Kusz, J. Chem. Crystallogr., 2010, 40, 201-206.

62 P. Silva, S. M. Vilela, J. P. Tome and F. A. Almeida Paz, Chem. Soc. Rev., 2015, 44, 6774-6803.

63 H. Furukawa, K. E. Cordova, M. O'Keeffe and O. M. Yaghi, Science, 2013, 341, 1230444.

64 A. G. Slater and A. I. Cooper, Science, 2015, 348, aaa8075.

65 M. N. Chretien, E. Heafey and J. C. Scaiano, Photochem. Photobiol., 2010, 86, 153-161.

66 M. Mitsui, K. Higashi, R. Takahashi, Y. Hirumi and K. Kobayashi, Photochem. Photobiol. Sci., 2014, 13, 11301136.

67 Y. F. Yao, M. S. Zhang, J. X. Shi, M. L. Gong, H. J. Zhang and Y. S. Yang, Mater. Lett., 2001, 48, 44-48.
1 